\title{
A note on dead-beat controllability of generalised Hammerstein systems
}

\author{
Dragan Nešić ${ }^{*}$ \\ Department of Systems Engineering, Australian National University, ACT 0200, Australia
}

Received 2 January 1996; revised 1 September 1996

\begin{abstract}
Necessary and sufficient conditions for dead-beat and complete controllability for a class of generalised Hammerstein systems are presented. Since the system's structure is very close to linear, only linear algebra is used for the controllability test. The test is very simple and easy to use. A closed-loop minimum-time dead-beat controller can be designed for a subclass of generalised Hammerstein systems using the Gröbner basis method.
\end{abstract}

Keywords: Dead-beat controllability; Complete controllability; Hammerstein systems

\section{Introduction}

Generalised Hammerstein systems may arise from identification techniques of the so-called block oriented models $[6,7]$. They represent a subclass of the class of input-output polynomial systems, very often referred to as NARMAX (nonlinear ARMAX) [6, 7]. Generalised Hammerstein systems can be regarded as a parallel connection of a simple Hammerstein system whose input nonlinearity is quadratic and a linear system, see Fig. 1. The output connection may be more complicated than the one presented in the figure but this is not crucial for our developments.

Although the structure of this class of models is very simple, it turns out to be adequate to model the dynamics of some practically important plants. For example, in [8] the model of a cement mill is identified as a generalised Hammerstein system. In the same paper, the author clains that his method can be used to identify models for some other milling processes. Another application of generalised Hammerstein models (multi-input) can be found in [1] where the model for

*Tel.: +61+6+2492456; e-mail: dragan.nesic@anu.edu.au.

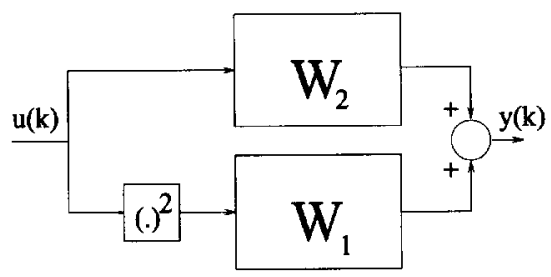

Fig. 1. Block diagram of a generalised Hammerstein system.

the cooling water circulation of a thermal power plant was identified in this form.

Roughly speaking, the property of dead-beat controllability shows our ability to achieve a desired operating regime of a system in finite time by means of actuators. Hence, dead-beat controllability is one of the most important properties of a controlled system, since it uncovers some fundamental limitations to the systems' performance. Therefore, dead-beat controllability tests are important tools in the analysis of control systems. The controller that transfers any initial state to the origin in minimum time is called a minimum-time dead-beat controller. It represents very often a simple and easy-to-use design option for 
a control engineer. The main disadvantage of deadbeat controllers is that they are not robust and may not perform well if the plant model is not correct.

Dead-beat control problems for linear systems have been studied thoroughly in the last 40 years [11] and a plethora of results have been reported. However, nonlinear dead-beat control has not received as much attention. There are several results in the literature that can be used to tackle the dead-beat problem for classes of NARMAX models. An interesting controllability test for discrete-time linear systems with positive controls was found in [5]. This result can be used to test controllability properties of simple Hammerstein systems [10], which are a subclass of NARMAX models. These systems are characterised by a series of a static nonlinearity and a linear dynamic block. Dead-beat controllers for simple Hammerstein systems were investigated in $[10,12]$.

In this paper, we present necessary and sufficient conditions for dead-beat controllability of generalised Hammerstein systems. We exploit the result on controllability of linear systems with positive controls [5] in the proof of our main result. The obtained deadbeat controllability test is very easy to use. Moreover, a complete controllability test easily follows from our method. We then present a closed-loop minimumtime dead-beat controller for a subclass of generalised Hammerstein systems which can be designed using the Gröbner basis method (Maple, Mathematica, etc.).

The paper is organised as follows. In Section 2 we present definitions, the class of systems that we consider and some results that are needed in the sequel. The main result is stated and proved in Section 3. Section 4 contains a design procedure for a closedloop minimum-time dead-beat controller of a class of generalised Hammerstein systems. The ideas and results are illustrated by some examples given in Section 5.

\section{Preliminaries}

We consider generalised Hammerstein systems of the form $[6,7]$ :

$$
\begin{aligned}
\left(\begin{array}{l}
x_{1}(k+1) \\
x_{2}(k+1)
\end{array}\right)= & \left(\begin{array}{cc}
F_{1} & 0 \\
0 & F_{2}
\end{array}\right)\left(\begin{array}{l}
x_{1}(k) \\
x_{2}(k)
\end{array}\right) \\
& +\left(\begin{array}{c}
g_{1} \\
0
\end{array}\right) u(k)+\left(\begin{array}{c}
0 \\
g_{2}
\end{array}\right) u^{2}(k),
\end{aligned}
$$

$$
\begin{aligned}
y(k)= & \left(\begin{array}{ll}
c_{1}^{\mathrm{T}} & c_{2}^{\mathrm{T}}
\end{array}\right)\left(\begin{array}{l}
x_{1}(k) \\
x_{2}(k)
\end{array}\right) \\
& +d_{0}+d_{1} u(k)+d_{2} u^{2}(k),
\end{aligned}
$$

where $x(k)=\left(x_{1}(k) x_{2}(k)\right)^{\mathrm{T}} \in \mathbb{R}^{n}$ is a state of the system at time $k$ and $u(k) \in \mathbb{R}$ is the control at time $k$. We also have $F_{1} \in \mathbb{R}^{n_{1} \times n_{1}}, F_{2} \in \mathbb{R}^{n_{2} \times n_{2}}, g_{1} \in \mathbb{R}^{n_{1} \times 1}, g_{2} \in$ $\mathbb{R}^{n_{2} \times 1}, x_{1}(k) \in \mathbb{R}^{n_{1}}$ and $x_{2}(k) \in \mathbb{R}^{n_{2}}$.

We denote a control sequence as $\mathscr{U}_{t}=\{u(0), u(1)$, $\ldots, u(t-1)\}, u(k) \in \mathbb{R}$. The state that is reached from $x(0)$ at time $t$ by applying the control sequence $\mathscr{U}_{t}$ is denoted as $x\left(t, x(0), \mathscr{U}_{t}\right)$. We need the following definitions:

Definition 1. The system (1) is dead-beat controllable if $\forall x(0) \in \mathbb{R}^{n}$ there exist finite $t=t(x(0))$ and a control sequence $\mathscr{U}_{t}$ such that $x\left(t, x(0), \mathscr{U}_{t}\right)=0$.

Definition 2. The system (1) is completely controllable if $\forall x(0), x^{*} \in \mathbb{R}^{n}$ there exist an integer $H=$ $H\left(x(0), x^{*}\right)$ and a finite control sequence $\mathscr{U}_{H}=$ $\{u(0), u(1), \ldots, u(H-1)\}$ such that the system is transferred from the state $x(0)$ to the state $x^{*}$ under the action of the sequence $\mathscr{U}_{H}$, that is $x\left(H, x(0), \mathscr{U}_{H}\right)=x^{*}$.

The following theorems play a crucial role for dead-beat controllability of generalised Hammerstein systems.

Theorem 1 (Evans and Murthy [5]). The system

$x(k+1)=A x(k)+b u(k)$

with $u \in\left[0,+\infty\left[\right.\right.$ is completely controllable on $\mathbb{R}^{n}$ if and only if

1. $\operatorname{rank}\left[b A b \ldots A^{n-1} b\right]=n$,

2. the matrix $A$ has no real positive or zero eigenvalues.

By slightly modifying Theorem 1 , we obtain conditions for dead-beat controllability [10].

Theorem 2. The system (2) with $u \in[0,+\infty[$ is deadbeat controllable on $\mathbb{R}^{n}$ if and only if

1. $\operatorname{rank}[\lambda I-A: b]=n, \forall \lambda \neq 0, \lambda \in \mathbb{C}$,

2. the matrix $A$ has no real positive eigenvalues.

Notice that the conditions of Theorem 1 are stronger than the conditions of Theorem 2. Indeed, in Theorem 2 the matrix $A$ is allowed to have zero 
eigenvalues (condition 2) and moreover zero modes do not have to be controllable (condition 1 ).

The following theorem is a consequence of Theorem 1. Its proof is contained in the proof of Theorem 1 in [5].

Theorem 3. If the second condition of Theorem 1 is satisfied, there exists a polynomial $C(\zeta)=$ $\sum_{i=0}^{N} c_{i} \zeta^{i}=0, c_{N}=1$ with $c_{i} \geqslant 0, \forall i=0,1, \ldots, N-1$ such that

$C(A)=0$.

Linear systems (2) with positive controls have an interesting propery: if the system (2) is completely controllable, there exists a uniform bound on the length of the control sequence in Definition 2. That is, there exists a number $H^{*}$ such that $H\left(x(0), x^{*}\right) \leqslant H^{*}, \forall x(0), x^{*} \in \mathbb{R}^{n}$. The same holds for dead-beat controllability of (2). We will see that the class of generalised Hammerstein systems (1) has the same property.

\section{Main result}

In this section we present necessary and sufficient conditions for dead-beat and complete controllability of generalised Hammerstein systems. It is shown that the system (1) is dead-beat (completely) controllable if and only if its subsystems

$x_{1}(k+1)=F_{1} x_{1}(k)+g_{1} u(k)$

and

$x_{2}(k+1)=F_{2} x_{2}(k)+g_{2} u^{2}(k)$

are dead-beat (completely) controllable.

The proof of the controllability result is based on the construction of a periodic open-loop controller. The obtained open-loop controller is theoretically important but it has all negative aspects of open-loop control schemes. Hence, a closed-loop controller is designed in the next section for a subclass of generalised Hammerstein systems.

Theorem 4. The system (1) is dead-beat controllable if and only if the following conditions are satisfied:

1. $\operatorname{rank}\left[I \lambda-F_{1}: g_{1}\right]=n_{1}, \forall \lambda \neq 0, \lambda \in \mathbb{C}$,

2. $\operatorname{rank}\left[I \lambda-F_{2}: g_{2}\right]=n_{2}, \forall \lambda \neq 0, \lambda \in \mathbb{C}$,

3. $F_{2}$ has no positive real eigenvalues.

Comment 1. The first condition of Theorem 4 means that the subsystem (3) is dead-beat controllable. The second and third conditions of Theorem 4 represent necessary and sufficient conditions for dead-beat controllability of the subsystem (4). It is obvious that dead-beat controllability does not require zero modes to be controllable.

Comment 2. Notice that if there are some zero eigenvalues of $F_{1}$ or $F_{2}$, we can find a nonsingular transformation $T$ such that

$\bar{F}_{i}=T^{-1} F_{i} T=\left(\begin{array}{cc}D_{11}^{i} & 0 \\ 0 & D_{22}^{i}\end{array}\right)$,

$\bar{g}_{i}=T^{-1} g_{i}, \quad i=1,2$

and $D_{22}^{i}$ is a nilpotent matrix. Assume that the degree of nilpotency of $D_{22}^{i}$ is $d_{i}$. Consider the state at step $k+1 \geqslant d_{i}$ :

$x_{i}(k+1)=\bar{F}_{i}^{k} x_{i}(0)+\sum_{l=0}^{k-1} \bar{F}_{i}^{k-l-1} \bar{g}_{i} u(l), \quad i=1,2$.

If we apply $u(l)=0, l=k-d_{i}, k-d_{i}+1, \ldots, k$, we have that $x_{i}(k+1)=\left(\begin{array}{ll}x^{\mathrm{T}} & 0\end{array}\right)^{\mathrm{T}}, i=1,2$ irrespective of the control sequence $u(l), l=0,1, \ldots, k-d_{i}-1$. Thus, there is no loss of generality if we concentrate just on situations when

$\operatorname{rank}\left[F_{i}-I \lambda: g_{i}\right]=n_{i}, \quad \forall \lambda \in \mathbb{C}, i=1,2$.

In other words, we assume that

1. $\operatorname{rank}\left[g_{1}: F_{1} g_{1}: \cdots: F_{1}^{n_{1}-1} g_{1}\right]=n_{1}$.

2. $\operatorname{rank}\left[g_{2}: F_{2} g_{2}: \cdots: F_{2}^{n_{2}-1} g_{2}\right]=n_{2}$.

3. $F_{2}$ has no zero or positive real eigenvalues.

Proof of Theorem 4. Necessity: Suppose that at least one of the conditions of Theorem 4 is violated. This implies that at least one of the subsystems (3) or (4) is not dead-beat controllable. Without loss of generality suppose that the subsystem (3) is not dead-beat controllable. From the definition of dead-beat controllability it follows that there exists an initial state $x_{1}^{*}(0) \in$ $\mathbb{R}^{n_{1}}$ for the subsystem (3) that cannot be driven to the origin in finite time. This implies that any initial states of the overall system (1) which is given by $\left(x^{*}(0)\right)^{\mathrm{T}}=$ $\left(\left(x_{1}^{*}(0)\right)^{\mathrm{T}} z^{\mathrm{T}}\right)^{\mathrm{T}}, z \in \mathbb{R}^{n_{2}}$ cannot be driven to the origin in finite time. Consequently, the overall system (1) is not dead-beat controllable by definition.

Sufficiency: In order to prove sufficiency we will consider special sequences of controls which can transfer any initial state of (1) to the origin if the conditions of theorem are satisfied.

Since the last two of the conditions in Comment 2 guarantee that the subsystems (4) are completely 
controllable, it is possible to find a sequence of controls $\mathscr{U}_{P}=\{u(0), u(1), \ldots, u(P-1)\}$ which yields $x_{2}(P)=0$ and $x_{1}(P) \in \mathbb{R}^{n_{1}}$. As a result, we assume without loss of generality that $x(0)=\left(\begin{array}{ll}x_{1}^{\mathrm{T}}(0) & 0\end{array}\right)^{\mathrm{T}}$.

Since $F_{2}$ has no positive or zero eigenvalues (see Comment 2), according to Theorem 3 the matrix $F_{2}$ satisfies a polynomial equation with real positive coefficients:

$$
\begin{aligned}
C\left(F_{2}\right)= & \sum_{i=0}^{i=N} c_{i} F_{2}^{i}=0, \\
& c_{i} \geqslant 0, \quad \forall i=0,1, \ldots, N .
\end{aligned}
$$

Consider now the following sequence of controls:

$$
\begin{aligned}
& u(0)= \pm \sqrt{c_{N}} v(0), \\
& u(1)= \pm \sqrt{c_{N-1}} v(0), \\
& u(2)= \pm \sqrt{c_{N-2}} v(0), \\
& \ldots \\
& u(N)= \pm \sqrt{c_{0}} v(0), \\
& u(N+1)= \pm \sqrt{c_{N}} v(1), \\
& \ldots \\
& u\left((N+1) n_{1}-1\right)= \pm \sqrt{c_{0}} v\left(n_{1}-1\right) .
\end{aligned}
$$

It is obvious that because of (5) the state of the subsystem (4) $x_{2}(k)$ is zeroed every $N+1$ steps irrespective of the values $v(k) \in \mathbb{R}, k=0,1, \ldots, n_{1}-1$. That is, $\forall v(k) \in \mathbb{R}$ we have that $x_{2}(N+1)=x_{2}(2(N+1))=$ $\cdots=x_{2}\left(n_{1}(N+1)\right)=0$.

Hence, we now consider if it is possible to zero the state of the subsystem $(3) x_{1}\left(n_{1}(N+1)\right)$ by using $v(k), k=0,1, \ldots, n_{1}-1$ if we start from any initial state $x_{1}(0) \in \mathbb{R}^{n_{1}}$. It is important to emphasize that the sign of control $u(k)$ and the values $v(k)$ in (7) can be arbitrarily assigned and it is this additional degree of freedom that we are exploiting in the proof.

We have:

$$
\begin{aligned}
x_{1}\left((N+1) n_{1}\right)= & \sum_{i=0}^{(N+1) n_{1}-1} F_{1}^{(N+1) n_{1}-1-i} g_{1} u(i) \\
& +F_{1}^{(N+1) n_{1}} x_{1}(0) .
\end{aligned}
$$

The control sequence (7) is now substituted in (7) and we want to specify the existence of appropriate signs and values $v(k), k=0,1, \ldots, n_{1}-1$ such that:

$$
\sum_{i=0}^{(N+1) n_{1}-1} F_{1}^{(N+1) n_{1}-1-i} g_{1} u(i)=-F_{1}^{(N+1) n_{1}} x_{1}(0) .
$$

We introduce the following vector functions:

$$
\begin{aligned}
& L_{0}=\sum_{i=0}^{i=N} F_{1}^{N-i} g_{1} \delta_{0, i} \\
& L_{1}=F_{1}^{N+1} \sum_{i=0}^{i=N} F_{1}^{N-i} g_{1} \delta_{1, i} \\
& \cdots \\
& L_{n_{1}-1}=F_{1}^{\left(n_{1}-1\right)(N+1)-1} \sum_{i=0}^{i=N} F_{1}^{N-i} g_{1} \delta_{n_{1}-1, i}
\end{aligned}
$$

where $\delta_{k, i}= \pm \sqrt{c_{N-i}}, \forall k=0,1, \ldots, n_{1}-1, i=$ $0,1, \ldots, N$. We can rewrite Eq. (8) as follows:

$$
\begin{aligned}
& -F_{1}^{(N+1) n_{1}} x_{1}(0) \\
& =\left[L_{0}: L_{1}: \cdots: L_{n_{1}-1}\right]\left(\begin{array}{c}
v(0) \\
v(1) \\
\cdots \\
v\left(n_{1}-1\right)
\end{array}\right) .
\end{aligned}
$$

If there exists a sequence of controls of the form (7) such that the matrix $\left[L_{0}: L_{1}: \cdots: L_{n_{1}-1}\right]$ is nonsingular then the system (1) is dead-beat controllable.

Because of non-singularity of $F_{2}$ there exists at least one $\delta_{k, i}>0$. Non-singularity of matrices $F_{1}$ and $F_{2}$ and controllability of the pair $\left(F_{1}, g_{1}\right)$ causes the vectors $L_{k}$ to have entries which are linear functions of $\delta_{k, i}, i=0,1, \ldots, N$. As a result, the determinant of $\left[L_{0}: L_{1}: \cdots: L_{n_{1}-1}\right]$ is a multi-linear function of $\delta_{k, i}$, which we denote as $p\left(\delta_{k, i}\right)$.

For any scalar valued affine function $l(y)=a y+b$, $a, b \in \mathbb{R}, a \neq 0$ in a scalar variable $y$, we have that if $l(y)=0$ then $l(-y) \neq 0$. This observation is exploited to select $\delta_{k, i}$ such that $p\left(\delta_{k, i}\right) \neq 0$.

Let us consider a multi-linear function with three $\delta_{k, i} \neq 0$, which we relabel as $\delta_{1}, \delta_{2}, \delta_{3}$. It is easy to check that any such function can be written in the following form:

$$
\begin{gathered}
\left(\left(K_{1} \delta_{1}+L_{1}\right) \delta_{2}+\left(K_{2} \delta_{1}+L_{2}\right)\right) \delta_{3} \\
+\left(K_{3} \delta_{1}+L_{3}\right) \delta_{2}+K_{4} \delta_{1}+L_{4},
\end{gathered}
$$

where $K_{i}, L_{i} \in \mathbb{R}$.

If $K_{1} \neq 0$, we can render $K_{1} \delta_{1}+L_{1} \neq 0$ by an appropriate choice of $\delta_{1}$. Moreover, with this choice of $\delta_{1}$ we can render $\left(K_{1} \delta_{1}+L_{1}\right) \delta_{2}+\left(K_{2} \delta_{1}+L_{2}\right)$ nonzero by choosing $\delta_{2}$ and finally the whole expression can be made nonzero by a choice of $\delta_{3}$. If $K_{1}=0$ but if $L_{1} \neq 0$ we can do the same, etc. By induction, we show that there is no combination of $\delta_{i}= \pm \sqrt{c_{i}}$ which renders (11) nonzero only if $K_{i}, L_{i}=0, i=1,2,3,4$ or $F_{2}$ is singular (that is, $\delta_{i}=0, i=1,2,3$ ). Since 
we assumed that $\delta_{i} \neq: 0$, it follows that either $F_{1}$ is singular or the pair $\left(F_{1}, g_{1}\right)$ is not controllable (e.g. $g_{1}=0$ ). Contradiction completes the proof. The argument can be carried out for a multi-linear function in any number of variables $\delta_{i, k}$ and hence conditions of Theorem 4 are sufficient for dead-beat controllability.

We emphasize that the result on complete controllability follows directly from the above given proof. Indeed, necessary and sufficient conditions for complete controllability of generalised Hammerstein systems (1) are easily checked to be the conditions $1-3$, given in Comment 2.

It is important to notice that although we have used a control sequence of non-minimal length in the proof, we did establish that there is a uniform bound on the number of steps necessary to perform dead-beat control.

\section{A minimum-time dead-beat controller}

In this section we present a method to design a closed-loop minimurn-time dead-beat controller for generalised Hammerstein systems, in the case when rank $F_{2}=1$. Actually w'e concentrate only on the situation when $F_{2}=f_{2} \in \mathbb{R}$. The method is based on the Gröbner basis method [4].

Roughly speaking, a Gröbner basis of a set of polynomials is a set of "simpler" polynomials. There exists an algorithm for the computation of a Gröbner basis which stops in finite time. For example, there are symbolic computation packages in Maple and Mathematica which compute Gröbner bases. We choose simplification rules (a monomial ordering) and the algorithm produces a Gröbner basis with respect to the specified rules. The Gröbner basis method generalises the Gauss' elimination algorithm for systems of linear algebraic equations to general polynomial equations.

The applications of Gröbner bases include the elimination problems, solving sets of polynomial equations, etc. [4]. For example, consider a set of polynomial equations:

$x+y-z^{3}=0, \quad x^{2}+y-z=0$,

$-x+y^{2}+z=0$.

The Gröbner basis of the polynomials $x+y-z^{3}, x^{2}+$ $y-z,-x+y^{2}+z$ with the lexicographic ordering $x>$ $y>z$ (the simplification rules) is $\left\{x+y-z^{3}, y-z^{3}+\right.$ $\left.y^{2}+z, 6 z-3 z^{3}-2 z^{4}-z^{9}+4 y z, 4 z-8 z^{3}+3 z^{5}+z^{11}\right\}$. Notice that the last polynomial in the Gröbner basis depends only on the variable $z$ and second and third polynomials on variables $y$ and $z$. Hence, we can find the solution of the equation $4 z-8 z^{3}+3 z^{5}+z^{11}=0$. If we substitute the obtained solutions into the second and third equations we obtain a set of polynomial equations in $y$ only which can again be solved, etc. Notice that the triangular structure of the Gröbner basis allows us to obtain solutions to the original set of polynomial equations in a much easier way. The relations between the solutions of the original set of polynomial equations and the solutions obtained from its Gröbner basis are summarised in the so-called extension and elimination theorems [4]. Due to space limitations we do not present any more details on the theory of Gröbner bases and refer to the first four chapters of [4] for the material that we need.

We assume that we have the information about the full state vector. This is not a restriction since if the pair $\left(\left(c_{1}^{\mathrm{T}} c_{2}^{\mathrm{T}}\right), \operatorname{diag}\left\{F_{1}, F_{2}\right\}\right)$ is observable, an observer can be constructed as follows:

$$
\begin{aligned}
z(k+1) & \\
= & \left(\begin{array}{cc}
F_{1} & 0 \\
0 & F_{2}
\end{array}\right) z(k)+\left(\begin{array}{c}
g_{1} \\
0
\end{array}\right) u(k)+\left(\begin{array}{c}
0 \\
g_{2}
\end{array}\right) u^{2}(k) \\
& +L\left(\left(c_{1}^{\mathrm{T}} c_{2}^{\mathrm{T}}\right) z(k)+d_{0}+d_{1} u(k)+d_{2} u^{2}(k)-y(k)\right),
\end{aligned}
$$

where $L$ is a column vector. The observer yields the error dynamics of the form:

$e(k+1)=\left[\left(\begin{array}{cc}F_{1} & 0 \\ 0 & F_{2}\end{array}\right)+L\left(\begin{array}{cc}c_{1}^{\mathrm{T}} & \left.c_{2}^{\mathbf{T}}\right)\end{array}\right)\right] e(k)$,

where $e(k)=z(k)-x(k)$. Because of the observability assumption, we can arbitrarily assign the error dynamics by appropriately choosing the vector $L$. Consequently, a dead-beat observer is designed by placing all the eigenvalues of the error dynamics matrix at the origin.

The main idea behind dead-beat control is that the sets $S_{0}, S_{1}, \ldots$ from which the origin can be reached in one, two, etc. time steps should be computed. It is not difficult to find an appropriate control law which maps all states in $S_{k+1}$ to $S_{k}, \forall k$, once the sets have been found. The computation of these sets can in principle be performed using the QEPCAD method (see [9]). It is shown below that the Gröbner basis method is also a natural tool to use in this setting if $\operatorname{rank} F_{2}=1$ (see Example 2). 
Let us rewrite the state equation in (1) as follows:

$x(k+1)=F x(k)+G_{1} u(k)+G_{2} u^{2}(k)$.

We also use shorter notation $x(k+1)=f(x(k), u(k))$, where $f(x, u)=F x+G_{1} u+G_{2} u^{2}$.

Consider the system (1). The state at time step $N+1$ can be represented as:

$$
\begin{aligned}
x(N+1)= & F^{N} x(0)+\sum_{i=0}^{N-1} F^{N-1-i} G_{1} u(i) \\
& +\sum_{i=0}^{N-1} F^{N-1-i} G_{2} u^{2}(i) .
\end{aligned}
$$

Let us compute the set of states that can be mapped to the origin in one step. of

Consider $N=1$ in the expression (12). If all rows

$F x(0)+G_{1} u(0)+G_{1} u^{2}(0)$

can be rendered zero by an appropriate choice of control $u(0)$ for a state $x(0)$, the state is in the set $S_{0}$. We denote the entries of $x(0)$ as $x_{1}, x_{2}, \ldots, x_{n}$. In order to compute the set $S_{0}$ we find the Gröbner basis of the rows of matrix (13) with the lex ordering $u(0)>x_{1}>x_{2}>\cdots>x_{n}$ [4]. The set of polynomials in the Gröbner basis $G B_{1}=\left\{c_{1}^{1}, \ldots, c_{M_{1}}^{l}\right\}$ which depend only on $x_{i}, i=1,2, \ldots, n$ defines the set $S_{0}$. In other words, we have

$S_{0}=\left\{x: c_{1}^{1}=0, \ldots, c_{M_{1}}^{1}=0\right\}$.

Of course, extension theorem [4] should be applied in order to check whether the set of polynomials $c_{i}^{1}$ completely defines the set $S_{0}$. It is interesting that for the examples that we solved, extension theorem was always satisfied.

Consider the expression (12) for $N=2$ and repeat the same procedure. We find the Gröbner basis of the rows of the matrix

$F^{2} x(0)+F G_{1} u(0)+F G_{1} u^{2}(0)+G_{1} u(1)+G_{2} u^{2}(1)$

with the lex ordering $u(0)>u(1)>x_{1}>x_{2}>$ $\cdots>x_{n}$ [4]. The polynomials in the basis $G B_{2}=$ $\left\{c_{1}^{2}, \ldots, c_{M_{2}}^{2}\right\}$ which depend only on $x_{1}, x_{2}, \ldots, x_{n}$ define the set $S_{1}$. That is, we have

$S_{1}=\left\{x: c_{1}^{2}=0, \ldots, c_{M_{2}}^{2}=0\right\}$.

We should again use extension theorem [4].

The procedure is continued until the step $N=n$, where $n$ is the order of the system. In this step there will be no polynomials in the Gröbner basis which depend only on $x_{i}, i=1,2, \ldots, n$. One of the polynomials depends, however, only on one control $u(n-1)$. Moreover, it turns out that it is a quadratic function in $u(n-1)$ and the discriminant of this polynomial $D(x)$ defines the set $S_{n-1}$ :

$S_{n-1}=\{x: D(x) \geqslant 0\}$.

Finally, if the system is dead-beat controllable we can prove that $S_{n}=\mathbb{R}^{n}$. Indeed, if we can prove that any initial state $x(0) \in \mathbb{R}^{n}$ can be transferred to $S_{n-1}$ in one step, then the state $x(0)$ can be transferred to the origin in $n+1$ time steps. Take one composition of discriminant $D$ (defining expression for the set $S_{n-1}$ ) with the map $f(x, u)$ and find which states can be mapped to $S_{n-1}$ in one step by choosing an appropriate control action. The composition is a quadratic function in $u$ with the coefficient that multiplies $u^{2}$ a positive constant. That is

$D(f(x, u))=a_{2} u^{2}+a_{1}(x) u+a_{0}(x), \quad a_{2}>0$.

Therefore, $\forall x \in \mathbb{R}^{n}$ there exists (perhaps very large) $u \in \mathbb{R}$ which renders $D(f(x, u))>0$. In other words, we can map any state in $\mathbb{R}^{n}$ to $S_{n-1}$ in one step. The above given procedure is carried out in Example 2 . Notice that the condition $S_{n}=\mathbb{R}^{n}$ could be proved also by using the expression (12) for $N=n+1$.

From the above given construction it follows that for dead-beat controllable generalised Hammerstein systems with $F_{2}=\left(f_{2}\right)$, the minimum dead-beat time is $n+1$ time steps. We emphasize that if the matrix $F_{2}$ is not scalar, equations of degree higher than 2 (in control $u$ ) occur in the above described procedure. This introduces an algebraic obstacle which can be resolved by using one of the quantifier elimination procedures in the first-order theory of real closed fields $[9,2,3]$. Indeed, a quantifier elimination algorithm such as QEPCAD $[2,3]$ should in general be used to design closed-loop minimum-time dead-beat controllers for generalised Hammerstein systems but computational complexity of the problem prevents us from using this method in general [9].

From the expressions for sets $S_{0}, S_{1}, \ldots$ it is not difficult to synthesize a closed-loop minimum-time deadbeat controller. The defining polynomials of $S_{k}, k=$ $0,1, \ldots, n-1$ are denoted as vectors

$C_{k}=\left(c_{1}^{k+1}(x) \cdots c_{M_{k+1}}^{k+1}(x)\right)^{\mathrm{T}}$.

The composition of two vector functions is denoted as $C_{k} \circ f=\left(c_{1}^{k+1}(f(x, u)) \cdots c_{M_{k+1}}^{k+1}(f(x, u))\right)^{\mathrm{T}}$, 
$u(k)=$ any real solution to

$$
\begin{cases}f=0 & \text { if } x(k) \in S_{0}, \\ C_{1} \circ f=0 & \text { if } x(k) \in S_{1}, \\ C_{2} \circ f=0 & \text { if } x(k) \in S_{2}, \\ \vdots & \vdots \\ C_{n-1} \circ f=0 & \text { if } x(k) \in S_{n-1}, \\ H(x) & \text { if } x(k) \in S_{n},\end{cases}
$$

where $H(x)$ is a function which yields

$D \circ f(x(k), H(x(k))) \geqslant 0$.

If the composition $D \circ f(x, u)=a_{2} u^{2}+a_{1}(x) u+a_{0}(x)$, one choice for $H(x)$ is

$H(x)=\frac{-a_{1}(x)+\sqrt{a_{1}^{2}(x)-4 a_{2} a_{0}(x)}}{2 a_{2}}+\delta, \quad \delta>0$.

In other words we have designed a family of closedloop minimum-time dead-beat controllers. The extra degrees of freedom can be used to shape the transient response.

\section{Examples}

Example 1. Consider the system (1) for which

$$
\begin{aligned}
& F_{1}=\left(\begin{array}{cc}
0 & 1 \\
-a_{0} & -a_{1}
\end{array}\right): \quad F_{2}=\left(-f_{2}\right), \\
& g_{1}=\left(\begin{array}{l}
0 \\
1
\end{array}\right), \quad g_{2}=(1) .
\end{aligned}
$$

Notice that $\left(F_{1}, g_{1}\right)$ and $\left(F_{2}, g_{2}\right)$ are controllable pairs. We assume that $f_{2}>0$ and that the matrix $F_{1}$ is not singular. Hence, all conditions of Theorem 4 are satisfied. $F_{2}=\left(-f_{2}\right)$ satisfies the equation

$F_{2}+f_{2} I=0$.

Consider the control sequence:

$u(0)=v(0)$,

$u(1)= \pm \sqrt{f_{2}} v(0)$

$u(2)=v(1)$,

$u(3)= \pm \sqrt{f_{2}} v(1)$.

Notice that we are not considering the most general sequence of controls, since we could in general have that $u(0)= \pm v(0), u(2)= \pm v(1)$. When we apply the control sequence to the system we obtain the following equation:

$$
\begin{aligned}
x_{1}(4)= & F_{1}^{4} x(0)+\left(F_{1}^{3} g_{1} \pm \sqrt{f_{2}} F_{1}^{2} g_{1}\right) v(0) \\
& +\left(F_{1} g_{1} \pm \sqrt{f_{2}} g_{1}\right) v(1) .
\end{aligned}
$$

If there exists a sequence of $\delta_{i}= \pm 1$ for which the matrix:

$$
\left[F_{1}^{3} g_{1}+\delta_{1} \sqrt{f_{1}} F_{1}^{2} g_{1}: F_{1} g_{1}+\delta_{2} \sqrt{f_{1}} g_{1}\right]
$$

is non-singular, the system is completely controllable. Since $p^{2}+a_{1} p+a_{0}$ is the characteristic polynomial of $F_{1}$, upon applying Cayley theorem we obtain that the determinant of the matrix (16)

$$
\begin{gathered}
\left(a_{1}^{2}-a_{0}-\delta_{1} t a_{1}\right)\left(-a_{1}+\delta_{2} t\right) \\
-2 a_{0} a_{1}+a_{1}^{3}-\delta_{1} t\left(a_{1}^{2}-a_{0}\right)
\end{gathered}
$$

which is equal to zero for all possible choices $\delta_{1}=$ $\pm 1, \delta_{2}= \pm 1$ if the following four equations are satisfied:

$$
\begin{aligned}
& \left(a_{1}^{2}-a_{0}-t a_{1}\right)\left(-a_{1}+t\right)-2 a_{0} a_{1} \\
& \quad+a_{1}^{3}-t\left(a_{1}^{2}-a_{0}\right)=0, \\
& \left(a_{1}^{2}-a_{0}+t a_{1}\right)\left(-a_{1}+t\right)-2 a_{0} a_{1} \\
& \quad+a_{1}^{3}+t\left(a_{1}^{2}-a_{0}\right)=0, \\
& \left(a_{1}^{2}-a_{0}+t a_{1}\right)\left(-a_{1}-t\right)-2 a_{0} a_{1} \\
& \quad+a_{1}^{3}+t\left(a_{1}^{2}-a_{0}\right)=0, \\
& \left(a_{1}^{2}-a_{0}-t a_{1}\right)\left(-a_{1}-t\right)-2 a_{0} a_{1} \\
& \quad+a_{1}^{3}-t\left(a_{1}^{2}-a_{0}\right)=0,
\end{aligned}
$$

where $t=\sqrt{f_{2}}$. Using the Gröbner basis method [4] (Maple software package) for polynomials in (17) with the lex ordering $a_{1}>a_{0}>t$, we obtain the Gröbner basis:

$\left\{a_{1} a_{0}, a_{1}^{2} t, a_{0} t, a_{1} t^{2}\right\}$.

In other words, Eqs. (17) are simultaneously satisfied if

$$
\begin{aligned}
& a_{1} a_{0}=0, \quad a_{1} t^{2}=0, \\
& a_{1}^{2} t=0, \quad a_{0} t=0
\end{aligned}
$$

and therefore at least one of the matrices $F_{1}$ or $F_{2}$ is singular. This contradicts the assumption that $F_{i}$ are non-singular. It is interesting that in this case we did not use the most general sequence of controls and still we could prove complete controllability. 
Example 2. We illustrate the method for the design of a closed-loop dead-beat controller which was presented in Section 4. Consider the system:

$$
\begin{aligned}
\left(\begin{array}{c}
x_{1}(k+1) \\
x_{2}(k+1) \\
x_{3}(k+1)
\end{array}\right)= & \left(\begin{array}{rrr}
1 & -2 & 0 \\
1 & -1 & 0 \\
0 & 0 & -1
\end{array}\right)\left(\begin{array}{l}
x_{1}(k) \\
x_{2}(k) \\
x_{3}(k)
\end{array}\right) \\
& +\left(\begin{array}{r}
3 \\
-1 \\
0
\end{array}\right) u(k)+\left(\begin{array}{l}
0 \\
0 \\
2
\end{array}\right) u^{2}(k) .
\end{aligned}
$$

The conditions of Theorem 4 are satisfied since

$\operatorname{rank}\left[g_{1}: F_{1} g_{1}\right]=\operatorname{rank}\left(\begin{array}{rr}3 & 5 \\ -1 & 4\end{array}\right)=2$,

$g_{2} \neq 0 \quad$ and $\quad f_{2}=-1<0$.

We compute first the set of states that can be transferred to the origin in one step. The entries of $x(0)$ are denoted as $x_{1}, x_{2}, \ldots, x_{n}$. If we compute the Gröbner basis of $\left\{x_{1}-2 x_{2}+3 u(0), x_{1}-x_{2}-u(0),-x_{3}+u^{2}(0)\right\}$ with the lex ordering $u(0)>x_{1}>x_{2}>x_{3}$ [4], we obtain $\left\{4 u(0)-x_{2}, 4 x_{1}-5 x_{2},-8 x_{3}+x_{2}^{2}\right\}$. Using the elimination and extension theorems in [4] we obtain

$S_{0}=\left\{x: 4 x_{1}-5 x_{2}=0\right.$ and $\left.-8 x_{3}+x_{2}^{2}=0\right\}$.

Find $x(2)$ as a function of $x_{i}(0), u(j), i=1,2,3$, $j=0,1$ and let $x(2)=0$. The Gröbner basis of $\left\{-x_{1}+5 u(0)+3 u(1),-x_{2}+4 u(0)-u(1), x_{3}-2 u^{2}(0)\right.$ $\left.+2 u^{2}(1)\right\}$ with the lex order $u(0)>u(1)>x_{1}>x_{2}>$ $x_{3}$ is $\left\{17 u(0)-x_{1}-3 x_{2}, 5 x_{2}+17 u(1)-4 x_{1}, 289 x_{3}+\right.$ $\left.30 x_{1}^{2}-92 x_{1} x_{2}+32 x_{2}^{2}\right\}$ and hence

$S_{1}=\left\{x: 289 x_{3}+30 x_{1}^{2}-92 x_{1} x_{2}+32 x 2^{2}=0\right\}$.

Having found $x(3)$ and letting $x(3)=0$, we compute the Gröbner basis of $\left\{-x_{1}+2 x_{2}-3 u(0)+5 u(1)+\right.$ $3 u(2),-x_{1}+x_{2}+u(0)+4 u(1)-u(2),-x_{3}+2 u^{2}(0)-$ $\left.2 u^{2}(1)+2 u^{2}(2)\right\}$ with the lex order $u(0)>u(1)>$ $u(2)>x_{1}>x_{2}>x_{3}$ :

$$
\begin{aligned}
& \left\{17 u(0)-17 u(2)-x_{1}-3 x_{2}, 5 x_{2}+17 u(1)-4 x_{1},\right. \\
& \quad-289 x_{3}+1156 u^{2}(2)-30 x_{1}^{2} \\
& \left.+92 x_{1} x_{2}+68 x_{1} u(2)-32 x_{2}^{2}+204 x_{2} u(2)\right\} .
\end{aligned}
$$

We see that the discriminant of the last polynomial in the basis must be positive, that is we find:

$$
\begin{aligned}
S_{2}=\{x & : 143344 x_{1}^{2}-397664 x_{1} x_{2}+189584 x_{2}^{2} \\
& \left.+1336336 x_{3} \geqslant 0\right\} .
\end{aligned}
$$

Take now one composition of the discriminant with (19):

$$
\begin{gathered}
-96873 x_{1}^{2}+378244 x_{1} x_{2}-544246 x_{1} u-274204 x_{2}^{2} \\
+302412 x_{2} u+2092127 u^{2}-668168 x_{3} .
\end{gathered}
$$

It is obvious that since the coefficient which multiplies $u^{2}$ is positive, we can render the expression (20) positive for any state in $\mathbb{R}^{3}$ and hence $S_{3}=\mathbb{R}^{3}$. We have constructively proved that the system is dead-beat controllable in 4 steps, by computing the sets $S_{k}, k=$ $0,1,2,3$. The design of a closed-loop minimum-time controller follows easily from the proof.

\section{Summary}

Necessary and sufficient conditions for deadbeat and complete controllability of generalised Hammerstein systems are presented. The conditions are very easy to check as opposed to the symbolic computation approaches proposed in [9]. A design method for closed-loop minimum-time dead-beat controllers for a subclass of generalised Hammerstein systems is described. The design relies on the Gröbner basis method, implemented in e.g. Maple.

\section{Acknowledgements}

The author wishes to acknowledge the funding of the activities of the Cooperative Research Centre for Robust and Adaptive Systems by the Australian Government under the Cooperative Research Centres Program. The author wishes to thank Professor I.M.Y. Mareels for many useful comments on this work.

\section{References}

[1] W. Bamberger and R. Isermann, Adaptive on-line steadystate optimisation of slow dynamic processes, Automatica 14 (1978) 223-230.

[2] G.E. Collins, Quantifier elimination for real closed fields by cylindrical algebraic decomposition, Lecture Notes in Computer Science, Vol. 33 (1975) 134-183.

[3] G.E. Collins and Hoon Hong, Partial cylindrical algebraic decomposition for quantifier elimination, J. Symbol. Comput. 12 (1991) 299- 138.

[4] D. Cox, J. Little and D. O'Shea, Ideals, Varieties and Algorithms (Springer, New York, 1992).

[5] M.E. Evans and D.N.P. Murthy, Controllability of discretetime systems with positive controls, IEEE Trans. Autom. Control 22 (1977) 942-945. 
[6] R. Haber and L. Kevic:sky, Identification of nonlinear dynamic systems, in: N.S. Rajbman, ed., Identification and System Parameter Estimation (North-Holland, Amsterdam, 1978) $79-127$.

[7] R. Haber and H. Unbehauen, Structure identification of nonlinear dynamic systems - a survey of input/output approaches, Automatica 26 (1990) 651-677.

[8] L. Keviczky, Nonlinear dynamic identification of a cement mill to be optimised, in: N.S. Rajbman, ed., Identification and System Parameter E:timation (North-Holland, Amsterdam, 1978) $1185-1195$.
[9] D. Nešic and I.M.Y. Mareels, Dead-beat controllability of polynomial systems: symbolic computation approaches, submitted for publication.

[10] D. Nešić and I.M.Y. Mareels, Dead-beat control of simple Hammerstein models, submitted for publication.

[11] J. O'Reilly, The linear time invariant time-optimal control problem -an overview, Automatica 17 (1981) 363-370.

[12] Q.-M. Zhu and K. Warwick, Dead-beat controller for a class of nonlinear systems, Control Theory Adv. Technol. 7 (1991) $101-116$. 